\title{
Performance comparison of bench-top, hyperspectral imaging and pocket near infrared spectrometers: the example of protein quantification in wheat flour
}

\author{
V. Baeten, ${ }^{*}$ J.A. Fernández Pierna, P. Vermeulen, B. Lecler, O. Minet, D. Zio and P. Dardenne \\ Walloon Agricultural Research Centre (CRA-W), Valorisation of Agricultural Products Department, Food and Feed Quality Unit, Henseval Building, \\ 24 Chaussée de Namur, 5030 Gembloux, Belgium. E-mail: v.baeten@cra.wallonie.be; FoodFeedQuality@cra.wallonie.be
}

There have been a number of recent developments in NIR technology which may prove to be significant in the near future. The overall objective of this work is to compare the performance of different NIR devices (i.e. bench-top, hyperspectral imaging and pocket NIR spectrometers) for the determination of a classical quality parameter, the protein content. In order to achieve this goal, several studies have been carried out with the different instrumentation on wheat flours (selected as a "homogeneous" product). These studies should determine if NIR hyperspectral imaging and NIR pocket instruments are as efficient as classical NIR bench-top instruments for predicting protein content.

\section{Introduction}

In Near Infrared (NIR) technology, there have been a number of developments recently which may prove to be significant. One recent development is, for instance, the combination of NIR spectroscopy and imaging technology to create hyperspectral technology, which allows spectral and spatial information to be obtained simultaneously. ${ }^{1,2}$ Other developments include different innovative technologies allowing the manufacture of compact and integrated handheld and pocket devices. The proper evaluation of these devices as well as the data transfer from bench-top spectrometers to handheld/ pocket or hyperspectral instruments is a continuous challenge. ${ }^{3}$

Furthermore, the prediction of protein content in cereals using NIR spectroscopy has been, for many years, well implemented in the agro-food sector. ${ }^{4}$ Classical bench-top NIR instruments are specially dedicated to this task, either at-line or on-line directly in the production chain. In order to illustrate this, Table 1 shows the performance of the

Table 1. Performances of the Belgian REQUASUD network (nine instruments) established in 1989 for wheat grain (Cereal Interlaboratory Study conducted in January 2017).

\begin{tabular}{|l|c|c|c|c|c|c|c|}
\hline & & \multicolumn{3}{|c|}{ Reference values } & \multicolumn{3}{|c|}{ NIR values } \\
\hline & Assigned value & Mean & sr & sR & Mean & sr & sR \\
\hline Protein (\% MS) & 12.89 & 13.05 & 0.12 & 0.47 & 12.8 & 0.24 & 0.44 \\
\hline
\end{tabular}

Legend: $\mathrm{sr}=$ repeatability; $\mathrm{sR}=$ reproducibility.

\section{Correspondence}

V. Baeten (v.baeten@cra.wallonie.be)

doi: 10.1255/nir2017.151

Citation: V. Baeten, J.A. Fernández Pierna, P. Vermeulen, B. Lecler, O. Minet,

D. Zio and P. Dardenne, "Performance comparison of bench-top, hyperspectral imaging and pocket near infrared spectrometers: the example of protein quantification in wheat flour", in Proc. $18^{\text {th }}$ Int. Conf. Near Infrared Spectrosc., Ed by S.B. Engelsen, K.M. Sørensen and F. van den Berg. IM Publications Open, Chichester, pp. 151-155 (2019). https://doi.org/10.1255/nir2017.151

\section{(C) 2019 The Authors}

This licence permits you to use, share, copy and redistribute the paper in any medium or any format provided that a full citation to the original paper is given, the use is not for commercial purposes and the paper is not changed in any way.

ISBN: 978-1-906715-27-4 
Belgium REQUASUD network since its establishment in 1989. ${ }^{5}$ The results demonstrate the high performance [in terms of repeatability (sr) and reproducibility (sR)] of the network. These results concern the inter-laboratory study performed in January 2017 regarding the determination of protein content by reference (combustion) method and classical bench-top NIR method in unground wheat grain.

The aim of the different studies conducted here is to show whether two of these recent NIR technology developments (pocket and hyperspectral imaging devices) can be considered as a reliable alternative to classical benchtop NIR spectrometers. The determination of the protein content on wheat flour, which is considered as a "homogeneous" product, has been selected as the experimental model. Wheat flour samples are easy to handle using classical NIR because the grinding process homogenises the product and protein content is a parameter that is stable over time. Two studies have been performed comparing bench-top instruments to hyperspectral imaging and to pocket instruments, respectively. In both cases, calibration models were developed using Partial Least Squares (PLS) for the common spectral range between instruments.

\section{Material and method Samples}

A set of 79 wheat flour samples was used for this study. The samples were collected in 2013, with 57 samples coming from Belgian mills and 22 from the Bureau Interprofessionnel des Etudes Analytiques (Bipea) in France, an independent association that provides testing programmes to laboratories.

\section{Reference analysis}

The Dumas combustion method was used to determine the total Nitrogen content, and therefore the crude protein content. The method involves the total combustion of samples under oxygen.

\section{Instrumentation and spectra acquisition}

Two classical bench-top NIR instruments working in reflection mode (Foss DS2500 and NIRSystems 5000), a NIR hyperspectral line-scan camera (BurgerMetrics) and two pocket NIR instruments (MicroNIR and $\mathrm{SCIO}$ ) were used. The bench-top Foss DS2500 (hereafter referred to as Foss-DS) operates in the $400-2498 \mathrm{~nm}$ range. The bench-top NIRSystem 5000 Autocup instrument equipped with a rotating sampling device (hereafter referred to as NIRSystem) operates in the 1100$2498 \mathrm{~nm}$ range. The NIR hyperspectral line-scan camera (BurgerMetrics, hereafter referred to as NIR-HSI) works in the $1100-2498 \mathrm{~nm}$ range. The pocket MicroNIR instrument from Viavi is active in the $920-1658 \mathrm{~nm}$ range. The pocket $\mathrm{SCIO}$ instrument from Consumer Physics has a range of $700-1100 \mathrm{~nm}$.

Figure 1 presents a NIR spectrum of wheat flour obtained with all the different instruments and, therefore, includes all the different spectral ranges used in this work.

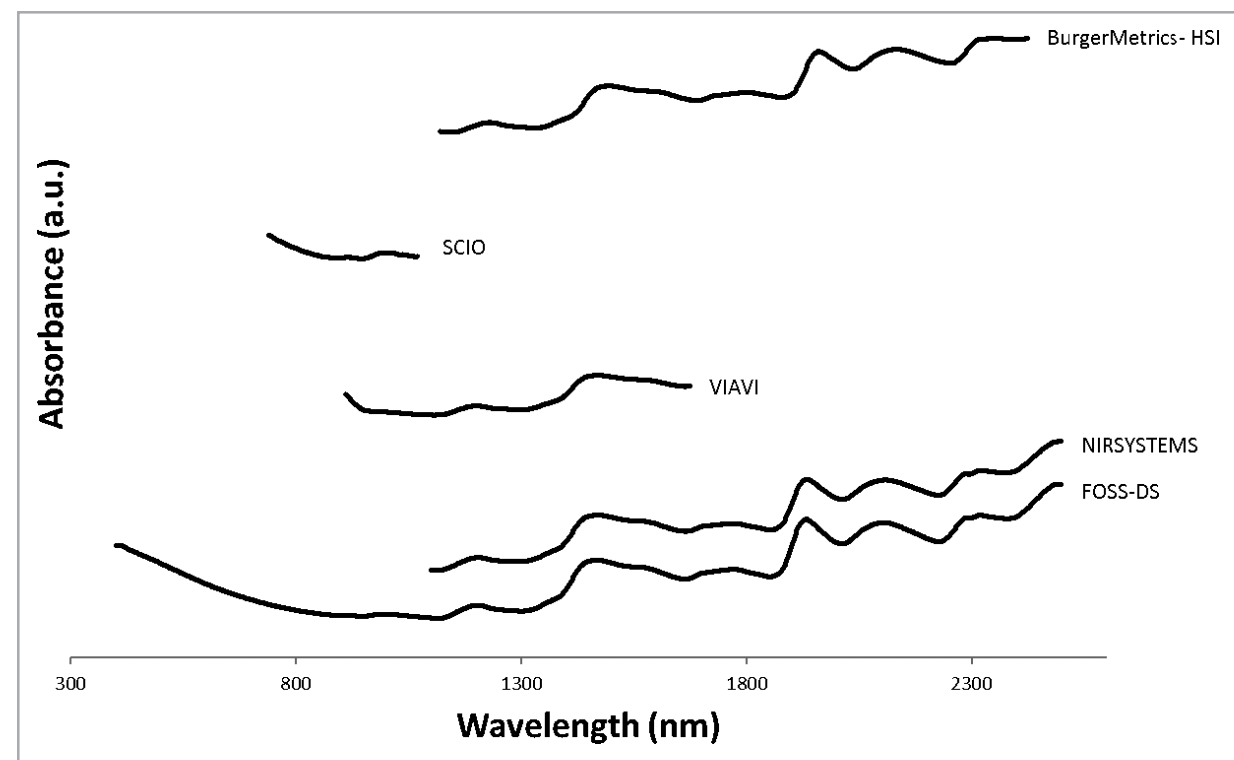

Figure 1. Spectral range comparison of the instruments used in these studies. 
For each instrument, the 79 samples of wheat flour were measured randomly, although always in the same order. Spectra acquisition was made in individual sealed cells using the two classical bench-top NIR instruments (Foss-DS and NIRSystem) and the NIR hyperspectral line-scan camera (NIR-HSI). The same sealed cups were used on the three different instruments. For the Foss-DS instrument, spectra were collected using the ISIscan Nova software across the original wavelength range. Each spectrum was the average of 32 scans performed on the sample and it was acquired in $66 \mathrm{~s}$. The sample area measured was about $2 \mathrm{~cm}^{2}$. For the NIRSystems instrument, spectra were collected using ISIscan software across the original wavelength range and each spectrum was the average of 32 scans performed on the sample. The sample area measured was about $2 \mathrm{~cm}^{2}$, and $57 \mathrm{~s}$ were needed to analyse a cell. For the NIR-HSI instrument, spectra acquisition was made using HyperPro software (BurgerMetrics SIA, Riga, Latvia). All the images consisted of 420 lines of 320 pixels acquired at 209 wavelength channels (1128-2425 nm) and 32 scans per image. A total of 2601 spectra $(51 \times 51$ pixels) corresponding to a sample area of $\pm 3 \mathrm{~cm}^{2}$ were selected from the centre of each image and averaged in order to obtain the mean spectra of each sample measured. In the configuration selected, the time required to analyse one sample was 28.8 s. For the $\mathrm{SClO}$ and Viavi, the time required to analyse one sample was about 30 s.

\section{Spectral data analysis}

The samples were split into two groups: a calibration set including the first 59 samples according to the measurement sequence, and a validation set with the remaining 20 samples. Both groups contained samples from different sources, wheat cultivars, growing conditions, harvesting regimes etc. The aim of this validation strategy was to check the stability over time of the models built.

The spectral data were processed using The Unscrambler software version 10.4 (CAMO).

\section{Results}

\section{Bench-top versus NIR-HSI}

The first study concerns the performance comparison of the classical bench-top NIR instruments (Foss-DS and NIRSystems) and the NIR-HSI line-scan camera for predicting protein content in wheat flour. As indicated, calibration models were developed using PLS for the full wavelength range of each individual instrument and for the common range between instruments (1100-2498 nm). The results showed that the NIR-HSI system worked as well as the classical bench-top NIR spectrometers when a common wavelength range was used, with a coefficient of determination of 0.99 for all instruments. Figure 2 presents the prediction results for the 20 samples of the independent validation set and the B-coefficients (also called regression coefficients) of the models built using only four terms for determination of protein content for the three instruments tested. Using four terms in the model for each instrument, the SEP is $0.23 \%, 0.13 \%$ and $0.37 \%$, respectively, for the Foss-DS, NIRSystems and NIR-HSI instrument. The profile of the B-coefficients of the three models built are quite similar. They demonstrate that the contributing variables are almost the same for the three instruments tested.

\section{Bench-top versus pocket instruments}

The second study concerns the comparison of the performances for the NIR-DS bench-top instrument to the pocket MicroNIR and SCIO NIR instruments. First, using NIR-DS data trimmed to the MicroNIR range (950$1650 \mathrm{~nm}$ ), a RMSEP of 0.23 was obtained analysing the validation set while a RMSEP of 0.42 was obtained for the MicroNIR data. Second, using NIR-DS data trimmed to the SCIO range $(700-1100 \mathrm{~nm})$, a RMSEP of 0.37 was obtained analysing the independent validation set while a RMSEP of 0.70 was obtained for the $\mathrm{SCIO}$ data. These results were obtained with models including only four terms. The differences between devices are indicative and should be confirmed by additional studies on different agro-food products. Table 2 presents the results, for the comparison of instruments, in terms of RMSEP for the validation set.

\section{Conclusions}

These studies were focused on the development of a model that can be applied on different types of instruments (global equation for protein content determination) and the right way to calibrate hyperspectral and pocket NIR spectrometers. The study concerning NIR hyperspectral imaging has proved that this kind of instru- 


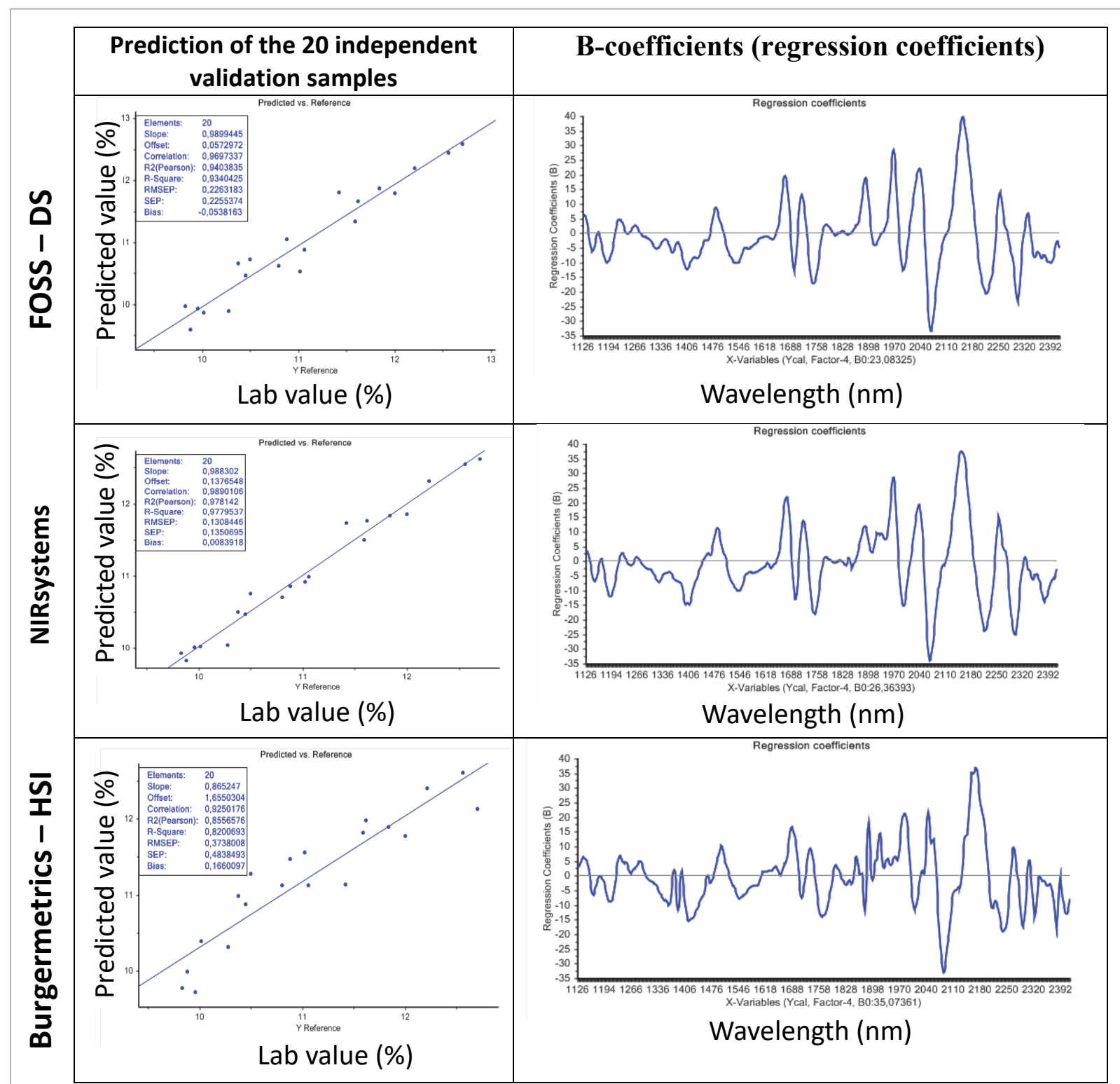

Figure 2. Comparison of the performance of three instruments limiting the number of terms to four. Left: prediction results for the validation set; right: B-coefficients for the three models.

ments, commonly focused on qualitative analysis, can also be used for classical quantitative analysis of agrofood products. In the case of pocket instruments, the performances still do not reach those obtained with

Table 2. Performance for the second study.

\begin{tabular}{|l|c|c|}
\hline Instrument & Range & RMSEP \\
\hline Foss-DS & $950-1650 \mathrm{~nm}$ & 0.23 \\
\hline Viavi-MicroNIR & $950-1650 \mathrm{~nm}$ & 0.42 \\
\hline Foss-DS & $700-1100 \mathrm{~nm}$ & 0.37 \\
\hline SCIO & $700-1100 \mathrm{~nm}$ & 0.70 \\
\hline
\end{tabular}

bench-top systems, but could be considered as a tool to give a fast, first indication of the quality of a product. Questions arise, as the calibration strategy has to be adapted for each type of instrument.

\section{Acknowledgements}

The authors thank the technical staff of the CRA-W: Nicaise Kayoka, Eric Fontaine, Sandrine Mauro, Nicolas Crasset, Stéphane Brichard and Arthur Dijon for their help in the realisation of the experiments presented as well as Ana María Morales, from the University of 
Seville, Spain for her collaboration in the hyperspectral study.

\section{References}

1. V. Baeten, J.A. Fernández Pierna, Ph. Vermeulen and P. Dardenne, "NIR hyperspectral imaging methods for quality and safety control of food and feed products: contributions to four European projects", NIR news 21(6), 10-13 (2010). https://doi.org/10.1255/ nirn.1200

2. L.M. Dale, A. Thewis, C. Boudry, I. Rotar, P. Dardenne, V. Baeten, J.A. Fernández Pierna, "Hyperspectral imaging applications in agriculture and agro-food product quality and safety control: A review", Appl. Spectrosc. Rev. 48, 142-159 (2013). https://doi.org/10.1080/05704928.2012.705800

3. J.A. Fernández Pierna, P. Vermeulen, B. Lecler, $\checkmark$. Baeten and P. Dardenne, "Calibration transfer from dispersive instruments to handheld spectrometers (MEMS)", Appl. Spectrosc. 64(6), 644-648 (2010). https://doi. org/10.1366/000370210791414353

4. P. Williams, "Grains and seeds", in Near-Infrared Spectroscopy in Food Science and Technology, Ed by Y. Ozaki, W.F. McClure and A.A. Christy. John Wiley \& Sons, Hoboken, USA (2007).

5. O. Minet, F. Ferber, L. Jacob, B. Lecler, R. Agneessens, T. Cugnon, V. Decruyenaere, V. Genot, S. Gofflot, E. Pitchugina, V. Planchon, M. Rennesson, G. Sinnaeve, J. Wavreille, P. Dardenne and V. Baeten, La Spectrométrie Proche Infrarouge: une Technologie Rapide, Précise et Écologique pour Déterminer la Composition et la qualité des Produits Agricoles et Alimentaires. ASBL Requasud, cellule de coordination, 32, Gembloux, Belgium (2016). 